Gazi Üniversitesi
Fen Bilimleri Dergisi
PART C: TASARIM VE TEKNOLOJI
dergipark.gov.tr/http-gujsc-gazi-edu-tr

\title{
Yeni Bir Tam Karmaşık Doğrusal Olmayan Adaptif Sonlu Darbe Cevaplı Filtre Algoritması
}

\author{
Engin Cemal MENGÜÇ \\ Niğde Ömer Halisdemir Üniversitesi, Mühendislik Fakültesi, Elektrik-Elektronik Mühendisliği Bölümü, 51245, Merkez/NíĞDE
}

\begin{abstract}
Öz
Makale Bilgisi

Başvuru: 20/05/2018

Düzeltme: 21/09/2018

Kabul: $25 / 10 / 2018$

Bu çalışmada, doğrusal olmayan karmaşık-değerli sinyaller için karmaşık-değerli doğrusal olmayan en küçük ortalama kurtosis (CNLMK) tabanlı yeni bir tam karmaşık doğrusal olmayan adaptif sonlu darbe cevaplı (FIR) filtre algoritması önerilmektedir. Önerilen doğrusal olmayan adaptif FIR filtre algoritmasının başarımı, karmaşık-değerli sinyallerin tahmini üzerinde test edilmiştir. Ayrıca önerilen algoritmanın başarımı, ortalama kare hata (MSE) ve tahmin kazancı cinsinden karmaşık-değerli doğrusal olmayan eğim düşüm (CNGD) algoritması ile karşılaştırılmıştır. Benzetim sonuçları önerilen CNLMK algoritmanın, CNGD algoritmasından daha üstün bir başarım sergilediğini açık bir şekilde ortaya koymuştur.
\end{abstract}

Anahtar Kelimeler

FIR filtre

CNLMK algoritmast

Tahmin

Karmaşılk-değerli sinyaller

Keywords

FIR filter

CNLMK algorithm

Prediction

Complex-valued signals

\section{A Novel Fully Complex Nonlinear Adaptive Finite Impulse Response Filter Algorithm}

\begin{abstract}
In this study, a new fully complex nonlinear adaptive finite impulse response (FIR) filter algorithm based on the complex-valued nonlinear least mean kurtosis (CNLMK) is proposed for nonlinear complex-valued signals. The performance of the proposed nonlinear adaptive FIR filter algorithm is assessed on the prediction of complex-valued signals. In addition, the performance of the proposed algorithm is compared with the complex-valued nonlinear gradient descent (CNGD) algorithm in terms of the mean square error (MSE) and the prediction gain. Simulation results clearly demonstrate that the proposed CNLMK algorithm outperforms the CNGD algorithm.
\end{abstract}

\section{GİRIŞ̧ (INTRODUCTION)}

Adaptif filtreler belirlenmiş bir maliyet fonksiyonunu kendi ağılık katsayılarını güncelleyerek minimize eden filtre tipleridir. Bu özelliklerinden dolayı birçok sinyal ișleme uygulamasında yaygın bir șekilde kullanılmaktadırlar [1-3]. Pratik uygulamalarda, genellikle sinyaller reel ve karmaşı-değerli sinyaller olmak üzere ikiye ayrılmaktadır. Reel-değerli sinyaller sadece genlik bilgisi taşırken, karmaşık-değerli sinyaller hem yön hem de şiddet bilgisine sahip olan sinyallerdir [2]. Literatürde yer alan çalışmalarda, genellikle sinyallerin genlik bilgisiyle ilgilenildiğinden tasarlanan adaptif filtre algoritmaları reel düzlemde tasarlanmıştır ve karmaşık-değerli sinyalleri doğrudan kullanılabilecek bir forma sahip değildirler [2]. Yön ve şiddet bilgisi içeren ve doğrudan gerçek dünya problemlerinde karşımıza çıkan karmaşık-değerli sinyal tiplerinin işlenebilmesi için reel düzlem yerine algoritmaların karmaşık düzlemde tasarlanmasına ihtiyaç vardır [2, 4-8]. Karmaşık-değerli sinyaller, sadece yön ve şiddet formunda değil aynı zamanda reel ve imajiner bileşenler formunda da ifade edilebilen sinyallerdir [2, 4-8]. Literatürde, karmaşık-değerli sinyallerin işlenebilmesi için karmaşık-değerli en küçük kare (complex-valued least mean squre (CLMS)) tabanlı algoritmalar tasarlanmıştır $[1-5,8]$. Bu algoritmaların tasarım süreci incelendiğinde, reel düzlemde ki tasarımlara benzer şekilde öncelikle minimize edilecek maliyet fonksiyonları karmaşık düzlemde ifade edilmektedir. Bu ifade edilen maliyet fonksiyonlarının, reel düzlemdeki maliyet fonksiyonlarından temel farkı karmaşık değişkenlerin reel değerli bir fonksiyonu olmasıdır. Karmaşık düzlemde tasarlanan CLMS tabanlı algoritmalar basit yapılarından dolayı oldukça popüler algoritmalardır fakat genel problemleri farklı 
gürültü tiplerinde yakınsama başarımlarının düşmesidir [7, 9]. Ayrıca küresel minimum noktaya yaklaşmayı her zaman garanti edemeyen adaptif filtre algoritma türleridir [7].

Son zamanlarda, adaptif filtrelerde başarımı artırmak için, yüksek dereceden istatistik tabanlı adaptif filtre algoritmaları önerilmiştir [9-20]. Bu algoritmaların içerisinde en küçük ortalama kurtosis (LMK) algoritması [9], hesaplama verimliliği ve farklı dağılımdaki gürültü sinyallerine karşı gürbüzlügü nedeniyle adaptif filtreleme problemlerinde ekin bir şekilde kullanılmaktadır [7, 9-16]. LMK algoritması [9], hata sinyalinin negatif kurtosis ile tanımlanmış maliyet fonksiyonunu en aza indirilmesine dayanmaktadır. Tanımlanan bu maliyet fonksiyonu, gürültü ile sistem uyumsuzluğunu istatistiksel olarak bir birinden ayırır ve bu sayede LMK algoritmasının farklı gürültü dağılımlarına karşı daha dayanıklı olmasını sağlar [9-16]. Ayrıca, LMK algoritmasının adım büyüklüğü yeterince küçük seçilirse, kurtosis maliyet fonksiyonu ile tanımlanan hata başarım yüzeyi küresel minimum noktaya sahip olur [9, 12-16]. LMK algoritmasının bu özellikleri son zamanlarda adaptif sinyal işleme alanında büyük bir ilgi uyandırmıştır. Literatürde yer alan çalışmalar [7, 9-16], LMK algoritmasının özellikle en küçük ortalama kare (LMS) tipi algoritmalardan daha iyi bir başarım sergilediğini göstermiştir. Fakat [9]'da önerilen LMK algoritması ilk olarak reel düzlemde tasarlandığından karmaşık-değerli sinyalleri işlemek için uygun değildir. Bu kapsamda ilk olarak Mengüç ve Acır [7]'de yer alan çalışmalarında, [9]'da önerilen LMK algoritmasının genişletilmiş versiyonu olan karmaşık-değerli LMK (CLMK) algoritmasını önermişlerdir. Yapılan çalışmada öncelikle maliyet fonksiyonu olarak hata sinyalinin kurtosis fonksiyonu karmaşık düzlemde tanımlanmış ve ardından CR analiz tekniği yardımıyla minimize edilmiştir. Yapılan bu çalışma [7], önerilen CLMK algoritmasının, karmaşık-değerli sistem kimlikleme problemleri için klasik CLMS algoritmasından daha iyi yakınsama ve kararlı durum başarımı gösterdiğini ortaya koymuştur. Önerilen algoritma [7] her ne kadar iyi bir başarım sergilese de doğrusal bir yapıya sahip olduğu için doğrusal olmayan karmaşık-değerli sinyallerin işlenebilmesi için uygun değildir.

Karmaşık-değerli sinyal işleme alanında, gerçek dünya sinyalleri hem doğrusal olmayan bir davranışa hem de geniş bir dinamiğe sahiptirler. Bu yüzden bu tip sinyallerin doğrusal sinyal işleme teknikleriyle işlenebilmesi oldukça zordur. Bu kapsamda literatürde [2, 21], CNGD tabanlı tam karmaşık doğrusal olmayan FIR filtre algoritması tasarlanmıştır. Fakat bu algoritma, CLMS algoritmasıyla benzer dezavantajlara sahiptir ve başarımının iyileştirilmesine ihtiyaç vardır.

Bu kapsamda, ilk defa bu çalışmada karmaşık-değerli doğrusal olmayan en küçük ortalama kurtosis (CNLMK) tabanlı yeni bir tam karmaşık doğrusal olmayan FIR filtre algoritması önerilmektedir. Bu çalışmada önerilen CNLMK algoritmasının, [7] ve [15]'de yer alan çalışmalardan temel farkı karmaşıkdeğerli doğrusal olmayan sinyallerin işlenebilmesi için doğrusal olmayan bir FIR filtre yapısına sahip olmasıdır. Önerilen algoritmanın başarımı, karmaşık-değerli sinyallerin tahmini üzerinde test edilmiş ve CNGD algoritması $[2,21]$ ile karşılaştırılmıştır. Yapılan bu çalışma, önerilen algoritmanın karmaşıkdeğerli sinyallerin tahmininde hem MSE hem de tahmin kazancı açısından başarımı dikkate değer bir şekilde artırdığını açık bir şekilde göstermektedir.

\section{TAM KARMAŞIK DOĞRUSAL OLMAYAN ADAPTIF FIR FILTRELERIN EĞITIMİ İÇIN CNLMK ALGORITMASININ TASARIMI (DESIGN OF CNLMK ALGORITHM FOR TRAINING FULLY COMPLEX ADAPTIVE FIR FILTERS}

Bu bölümde, CNLMK algoritmasının tasarım süreci sunulmaktadır. Şekil 1'de tam karmaşık doğrusal olmayan adaptif FIR filtrenin blok diyagramı yer almaktadır. Burada filtrenin çıkışı aşağıdaki gibi tanımlanır:

$y(k)=\Phi\left(\mathbf{w}^{H}(k) \mathbf{x}(k)\right)$

burada $\Phi, \quad H, \quad \mathbf{w}(k)=[w(k), w(k-1), \ldots, w(k-M)]^{T} \quad$ ve $\quad \mathbf{x}(k)=[x(k), x(k-1), \ldots, x(k-M)]^{T}$ sirasiyla karmaşık-değerli doğrusal olmayan fonksiyonu, eşlenik transpozu, filtre ağırlık vektörünü ve filtre giriş vektörünü tanımlamaktadır. Ayrıca $M$ ifadesi doğrusal olmayan filtrenin derecesini, $T$ ise transpozu temsil etmektedir. Karmaşık-değerli hata sinyali ise Denklem (2.2)'de tanımlanmaktadır. 
$e(k)=d(k)-y(k)=d(k)-\Phi\left(\mathbf{w}^{H}(k) \mathbf{x}(k)\right)$

burada $d(k)$ beklenen sinyali temsil etmektedir.

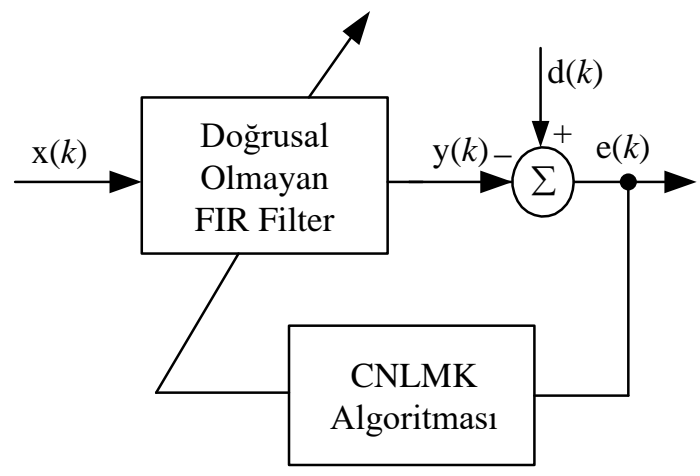

Şekil 1. Tam karmaşık doğrusal olmayan adaptif FIR filtrenin blok diyagramı

Yapılan bu tanımlamaların ardından, hata fonksiyonun kurtosisi ile tanımlanan maliyet fonksiyonu [7] karmaşık düzlemde aşağıdaki gibi oluşturulur:

$J(k)=3 E^{2}\left\{e(k) e^{*}(k)\right\}-E\left\{e(k) e^{*}(k) e(k) e^{*}(k)\right\}$

burada $E$ beklenen değeri temsil eder. Buradaki amacımız, tanımlanan bu maliyet fonksiyonunu karmaşıkdeğerli filtre ağırlık vektörü $\mathbf{w}(k)$ ' ya göre minimize ederek en iyi ağırlık vektörü olan $\mathbf{w}_{\mathrm{o}}(k)$ 'yı elde etmektir. Fakat maliyet fonksiyonu $J(k)$, karmaşık değişkenlerin reel-değerli bir fonksiyonu olduğundan Cauchy-Riemann koşullarını sağlamaz ve bu yüzden doğrudan karmaşık düzlemde kısmi türevi alınamaz. $\mathrm{Bu}$ problemin üstesinden gelebilmek için literatürde yaygın kullanılan CR analiz tekniğinin (aynı zamanda Wirtinger analiz olarak da bilinen) kullanılması gerekir $[2,9]$. CR analiz sayesinde bu tip fonksiyonların hem $\mathbf{w}(k)$ hem de $\mathbf{w}^{*}(k)$ 'ya göre kısmi türevleri alınabilir [2]. CR analiz tekniği için detaylı bilgiye [2, 7]'den ulaşılabilir. CR analiz kullanılarak, Denklem (2.3)'de tanımlanan maliyet fonksiyonu $J(k)$ 'nın $\mathbf{w}^{*}(k)$ ’ya göre türevi aşağıdaki gibi ifade edilir:

$\nabla J_{C L M K}=\frac{\partial J_{C L M K}}{\partial \mathbf{w}^{*}(k)}=6 E\left\{e(k) e^{*}(k)\right\} \frac{\partial E\left\{e(k) e^{*}(k)\right\}}{\partial \mathbf{w}^{*}(k)}-\frac{\partial E\left\{e(k) e^{*}(k) e(k) e^{*}(k)\right\}}{\partial \mathbf{w}^{*}(k)}$

Denklem (2.4)'de yer alan kısmi türevlerin beklenen değerleri [4, 5]'deki çalışmalara benzer şekilde, stokastik yaklaşım kullanılarak anlık değerleriyle yer değiştirilir. Böylece maliyet fonksiyonu $J(k)$ 'nın stokastik yaklaşımı $\hat{\nabla} J_{C L M K}$ aşağıdaki gibi yeniden ifade edilir:

$\hat{\nabla} J_{C L M K}=6 E\left\{e(k) e^{*}(k)\right\} \frac{\partial\left(e(k) e^{*}(k)\right)}{\partial \mathbf{w}^{*}(k)}-\frac{\partial\left(e(k) e^{*}(k) e(k) e^{*}(k)\right)}{\partial \mathbf{w}^{*}(k)}$. 
Denklem (2.5)'de ki ifade çözüldüğünde ise:

$$
\hat{\nabla} J_{C L M K}=-4\left(3 E\left\{e(k) e^{*}(k)\right\}-e^{*}(k) e(k)\right) e^{*}(k) \Phi^{\prime}(k) \mathbf{x}(k)
$$

ifadesi elde edilir. Burada $\Phi^{\prime}(k)=\Phi^{\prime}\left(\mathbf{w}^{H}(k) \mathbf{x}(k)\right)$ tam karmaşık doğrusal olmayan fonksiyonun türevini ifade ederken, $E\left\{e(k) e^{*}(k)\right\}$ niceliği hata sinyalinin varyansı $\sigma_{e}^{2}(k)$ 'yı temsil eder. Gerçek zamanlı uygulamalarda ise $E\left\{e(k) e^{*}(k)\right\}=\sigma_{e}^{2}(k)$ niceliğinin Denklem (2.7)'deki ifade kullanılarak anlık olarak kestirilmesi gerekmektedir [7, 9].

$$
\sigma_{e}^{2}(k)=\beta \sigma_{e}^{2}(k-1)+e(k) e^{*}(k), 0<\beta<1
$$

burada $\beta$ unutma faktörünü temsil etmektedir ve bu parametrenin 0 ile 1 arasında bir değere kurulmas 1 gerekmektedir.

Sonuç olarak, önerilen CNLMK algoritmasının ağrılık vektörü güncelleme kuralı aşağıdaki gibi elde edilmiştir.

$$
\begin{aligned}
\mathbf{w}(k+1) & =\mathbf{w}(k)+\mu\left(-\hat{\nabla} J_{C L M K}\right) \\
& =\mathbf{w}(k)+\mu\left(3 \sigma_{e}^{2}(k)-e^{*}(k) e(k)\right) e^{*}(k) \Phi^{\prime}(k) \mathbf{x}(k)
\end{aligned}
$$

burada adım büyüklüğü $\mu$ küçük bir değer seçilmesi durumunda, önerilen algoritma en iyi çözüme yakınsamayı garanti eder [7, 9]. Ayrıca belirtmek gerekir ki Denklem (2.6)'da yer alan 4 sabiti $\mu$ 'nün içerisine yerleştirilmiştir. Hem adım büyüklüğü $\mu$ hem de unutma faktörü $\beta$ algoritmanın yakınsama hızı ile ortalama kare hata arasındaki dengeyi kontrol eder. Unutma faktörünün her ne kadar 0 ile 1 arasında seçilmesi gerekse de, pratikte bu değerin 1'e yakın bir değere kurulması gerekir. Önerilen CNLMK algoritmasının sözde kodu adım adım Tablo 1'de sunulmuştur.

Tablo1. CNLMK Algoritmasinin Sözde Kodu

\section{Parametreler:}

$\mu<<1, \mu \in \mathfrak{R}^{+}$ve $0<\beta<1$.

$\mathbf{w}(0)=\mathbf{0}$ ve $M$ doğrusal olmayan FIR filtrenin derecesi

Veri: $\left\{x_{k}, d_{k}\right\}_{k=1}^{K}$

\section{Algoritma Döngüsü:}

$$
\begin{aligned}
& y(k)=\Phi\left(\mathbf{w}^{H}(k) \mathbf{x}(k)\right) \\
& e(k)=d(k)-y(k) \\
& \sigma_{e}^{2}(k)=\beta \sigma_{e}^{2}(k-1)+e(k) e^{*}(k) \\
& \mathbf{w}(k+1)=\mathbf{w}(k)+\mu\left(3 \sigma_{e}^{2}(k)-e^{*}(k) e(k)\right) e^{*}(k) \Phi^{\prime}(k) \mathbf{x}(k)
\end{aligned}
$$




\section{BENZETIM SONUÇLARI VE TARTIŞMA (SIMULATION RESULTS AND DISCUSSION)}

Bu çalışmada, önerilen CNLMK algoritmasının başarımı tahmin problemi üzerinde test edilmiştir. Bütün benzetimlerde doğrusal olmayan FIR filtrenin derecesi 4 olarak seçilmiştir. Ayrıca, FIR filtrenin doğrusal olmayan fonksiyonu $\Phi$, karmaşık-değerli hiperbolik tanjant fonksiyonu olarak seçilmiştir. Önerilen CNLMK algoritmasının başarımı CNGD algoritması [2,6] ile karşılaştırılmıştır. Yapılan çalışmalarda, önerilen algoritmanın adım büyüklüğü ve unutma faktörü sırasıyla $\mu=5 \times 10^{-3}$ ve $\beta=0.995$ olarak seçilmiştir. Öte yandan iyi bir başarım elde etmek için CNGD algoritmasının adım büyüklüğü $\mu=0.01$ olarak seçilmiştir. Başarım ölçütü olarak ise Denklem (3.1)'de verilen standart tahmin kazancı $R_{p}$ [2] ve MSE ifadeleri kullanılmıştır. Çalışmada sunulan benzetim sonuçları, bağımsız 500 deneyin ortalaması alınarak elde edilmiştir.

$$
R_{p}=10 \log _{10}\left(\sigma_{d}^{2} / \sigma_{e}^{2}\right)[d B]
$$

burada $\sigma_{d}^{2}$ ve $\sigma_{e}^{2}$ sırasıyla giriş ve anlık hata sinyallerinin varyansını temsil etmektedir.

Yapılan çalışmada, literatürde yaygın bir şekilde kullanılan karmaşık-değerli sentetik sinyaller ve gerçek dünya sinyali olan karmaşık-değerli radar sinyali kullanılmıştır. Bu sinyallere ait genel bilgilere ve matematiksel ifadelere aşağıda yer verilmiştir.

Çalışmada kullanılan ilk sinyal Denklem (3.2)'de verilen karmaşık-değerli otoregresif (autoregressive (AR)) süreçtir [2].

$$
d(k)=1.79 d(k-1)-1.85 d(k-2)+1.27 d(k-3)-0.41 d(k-4)+n(k) .
$$

Kullanılan ikinci sinyal, Denklem (3.3)'de verilen karmaş1k-değerli otoregresif yürüyen ortalama (auto regressive moving average (ARMA)) sürecidir [2].

$d(k)=0.7 d(k-1)+2 n(k)+0.5 n^{*}(k)+n(k-1)+0.9 n^{*}(k-1)$

Denklem (3.2) ve (3.3)'de, $n(k)$ ifadesi karmaşık-değerli sıfır ortalamalı birim varyansa sahip beyaz gürültüdür. Ayrıca bu sinyalin reel ve imajiner bileşenleri bir birinden bağımsızdır.

Üçüncü sinyal olarak ise Denklem (3.4)'de verilen karmaşık-değerli doğrusal olmayan Ikeda sinyali [2] benzetimlerde kullanılmıştır.

$$
\begin{aligned}
& t(k)=0.4-\left(6 /\left(1+x^{2}(k)+y^{2}(k)\right)\right) x(k+1)=1+u(x(k) \cos [t(k)]-y(k) \sin [t(k)]) \\
& y(k+1)=u(x(k) \sin [t(k)]+y(k) \cos [t(k)])
\end{aligned}
$$

burada $u=0.8$.

Son olarak ise yapılan çalışmada, karmaşık-değerli gerçek dünya sinyali olan radar sinyali [22] kullanılmıştır. Radar sinyali [22]'de verilen web sitesinden elde edilmiştir. 
Şekil 2, 3, 4 ve 5'de sırasılla benzetimde kullanılan karmaşık-değerli AR, ARMA, Ikeda ve Radar sinyallerinin reel ve imajiner eksen üzerindeki çizimleri yer almaktadır. Şekil 2, 3, 4 ve 5'den görüldüğü üzere bütün sinyaller reel ve imajiner eksen üzerinde farklı şekilde dağılımlara sahiptirler. Tablo 2'de ise benzetimde kullanılan 4 farklı sinyal içinde CNLMK ve CNGD algoritmalarının standart tahmin kazançları desibel (dB) cinsinden sunulmuştur. Tablo 2'den gözlemlenebildiği üzere, önerilen CNLMK algoritması, karmaşık-değerli AR, ARMA, Ikeda ve Radar sinyallerinin tahmininde CNGD algoritmasına kıyasla daha yüksek bir $R_{p}(\mathrm{~dB})$ kazancı sağlamıştır. Önerilen algoritmanın bu yüksek başarımının temel sebebi ise Denklem (2.3)'de tanımlanan maliyet fonksiyonun yüksek dereceden istatistiksel bilgileri içermesidir. Bilindiği üzere, CNGD algoritmasının maliyet fonksiyonu sadece ikinci dereceden istatistiksel bilgileri içerir ve buda başarımı önemli ölçüde kısıtlar.

Önerilen CNLMK algoritmanın başarımı sadece tahmin kazancı cinsinden değil aynı zaman da MSE (dB) başarımı cinsinden de ölçülmüştür. MSE (dB) başarımın ölçülmesinin temel sebebi, önerilen algoritmanın hem yakınsama hızının hem de kararlı durum hatasının açık bir şekilde gözlemlenebilmesi içindir. Şekil 6, 7 ve 8'de sirasıyla benzetimlerde kullanılan karmaşık-değerli AR, ARMA ve Ikeda sinyalleri için algoritmaların MSE (dB) başarımları sunulmaktadır. Şekil 6, 7 ve 8'de, önerilen CNLMK algoritması hem yakınsama hızı hem de kararlı durum MSE (dB) hatası anlamında CNGD algoritmasından daha üstün bir başarım sergilediği açık bir şekilde gözlemlenmektedir. Ayrıca belirtmemiz gerekir ki, karmaşık-değerli radar sinyali tek tur bir ölçüm olduğundan dolayı algoritmaların MSE (dB) başarımları benzetimlerde sunulmamıştır.

Sonuç olarak, yüksek dereceden istatistiksel bilgileri yapısında bulunduran CNLMK algoritması çeşitli karmaşı-değerli sinyallerin tahmininde, ikinci dereceden istatistiksel bilgilerle çalışan CNGD algoritmasına göre başarımı ciddi bir şekilde artırmıştır. Bu çalışmada elde edilen sonuçlar; önerilen algoritmanın, doğrusal olmayan adaptif sinyal işleme alanına önemli katkılar sağlayacağını açık bir şekilde göstermiştir.

Tablo 2. Karmaşık-değerli AR, ARMA, Ikeda ve Radar sinyalleri için CNLMK ve CNGD algoritmalarının tahmin kazancı başarımları

\begin{tabular}{|l|l|l|}
\hline \multirow{2}{*}{ Karmaşık-Değerli Sinyaller } & \multicolumn{2}{|l|}{ Algoritmaların $R_{p}(\mathrm{~dB})$ Tahmin Kazançları } \\
\cline { 2 - 3 } & CNGD & CNLMK \\
\hline AR & 3.2208 & $\mathbf{5 . 5 0 0 1}$ \\
\hline ARMA & 3.3999 & $\mathbf{4 . 8 4 9 1}$ \\
\hline Ikeda & 1.9821 & $\mathbf{2 . 6 0 4 0}$ \\
\hline Radar & 14.5142 & $\mathbf{1 5 . 9 7 6 0}$ \\
\hline
\end{tabular}




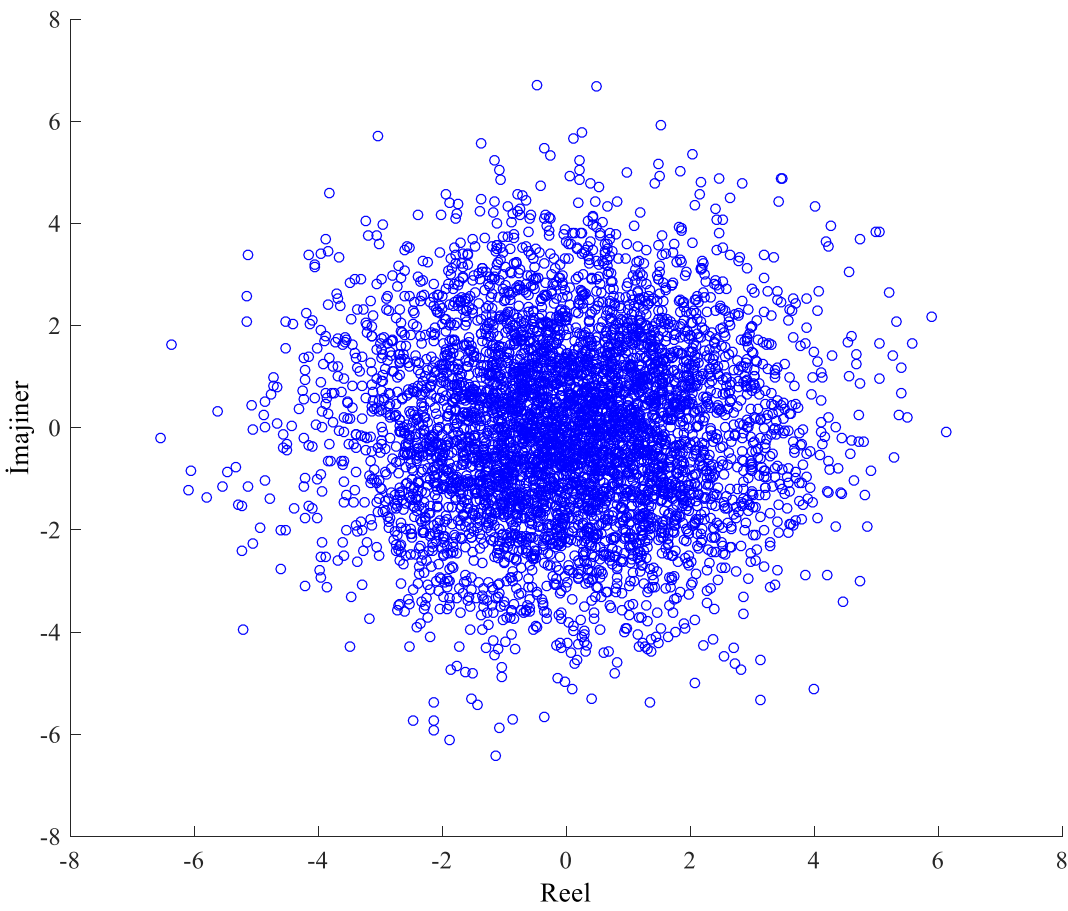

Şekil 2. Benzetimde kullanılan karmaşık-değerli AR sinyalinin reel ve imajiner eksen üzerindeki gösterimi

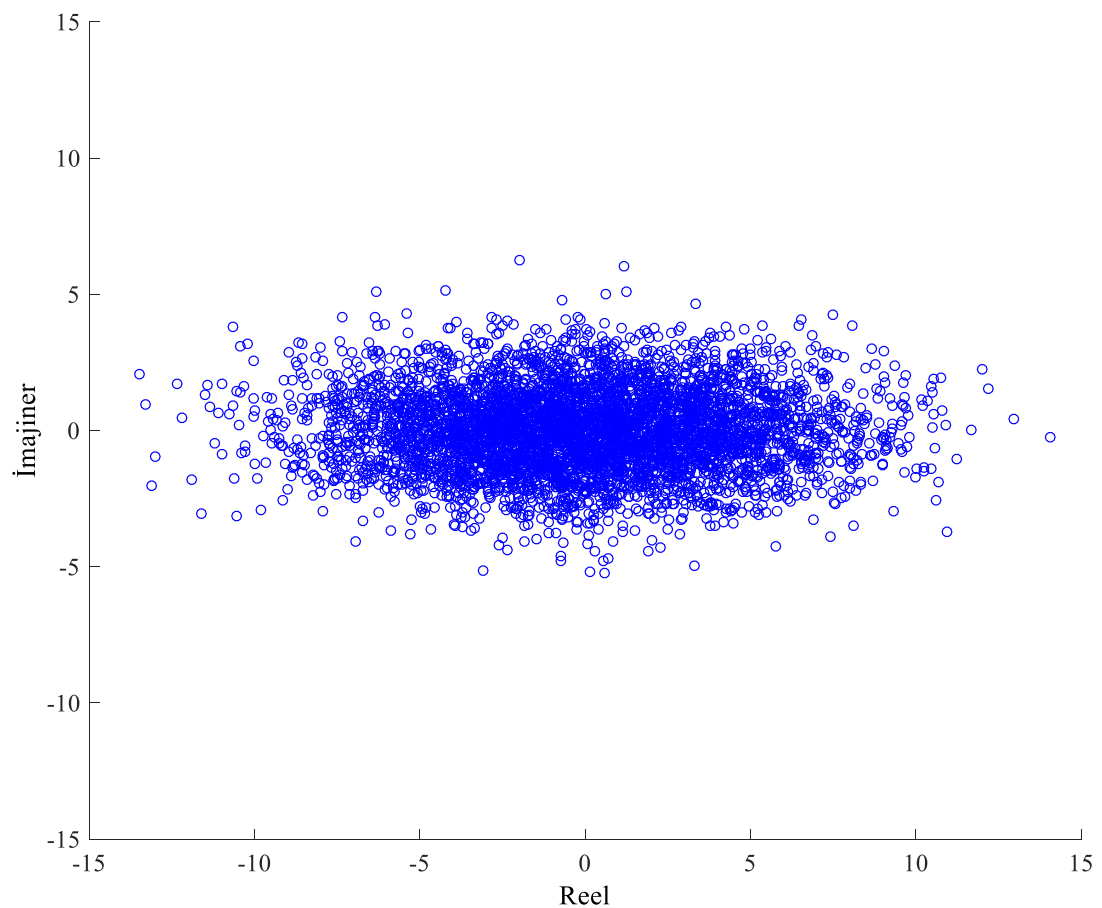

Şekil 3. Benzetimde kullanılan karmaşık-değerli ARMA sinyalinin reel ve imajiner eksen üzerindeki gösterimi 


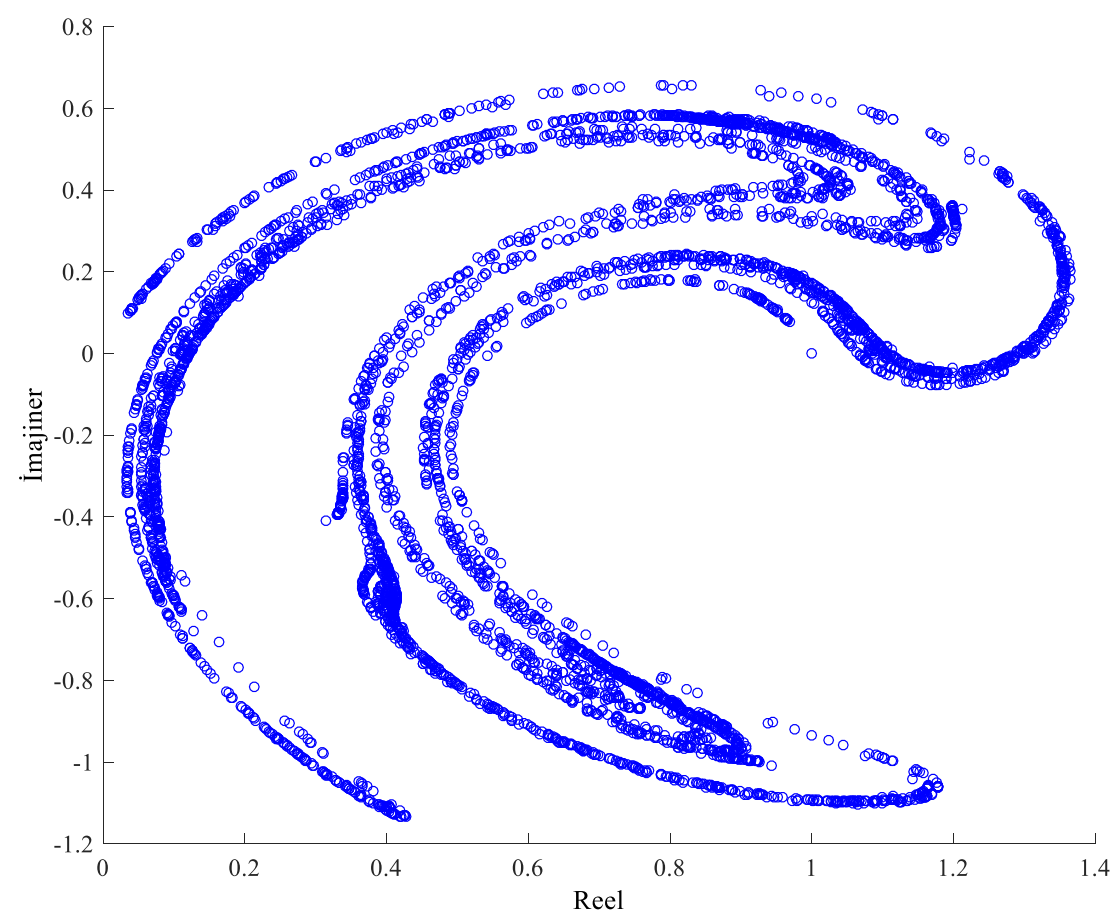

Şekil 4. Benzetimde kullanılan karmaşık-değerli Ikeda sinyalinin reel ve imajiner eksen üzerindeki gösterimi

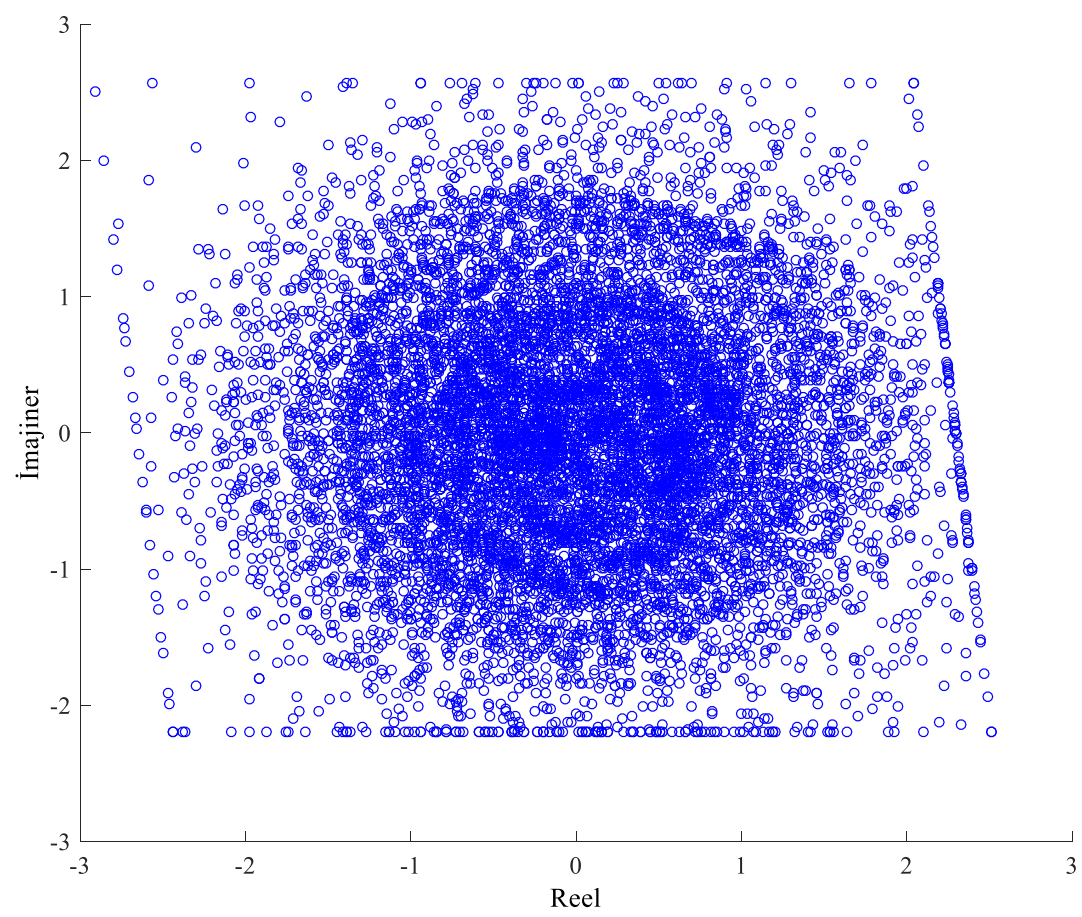

Şekil 5. Benzetimde kullanılan karmaşık-değerli Radar sinyalinin reel ve imajiner eksen üzerindeki gösterimi 


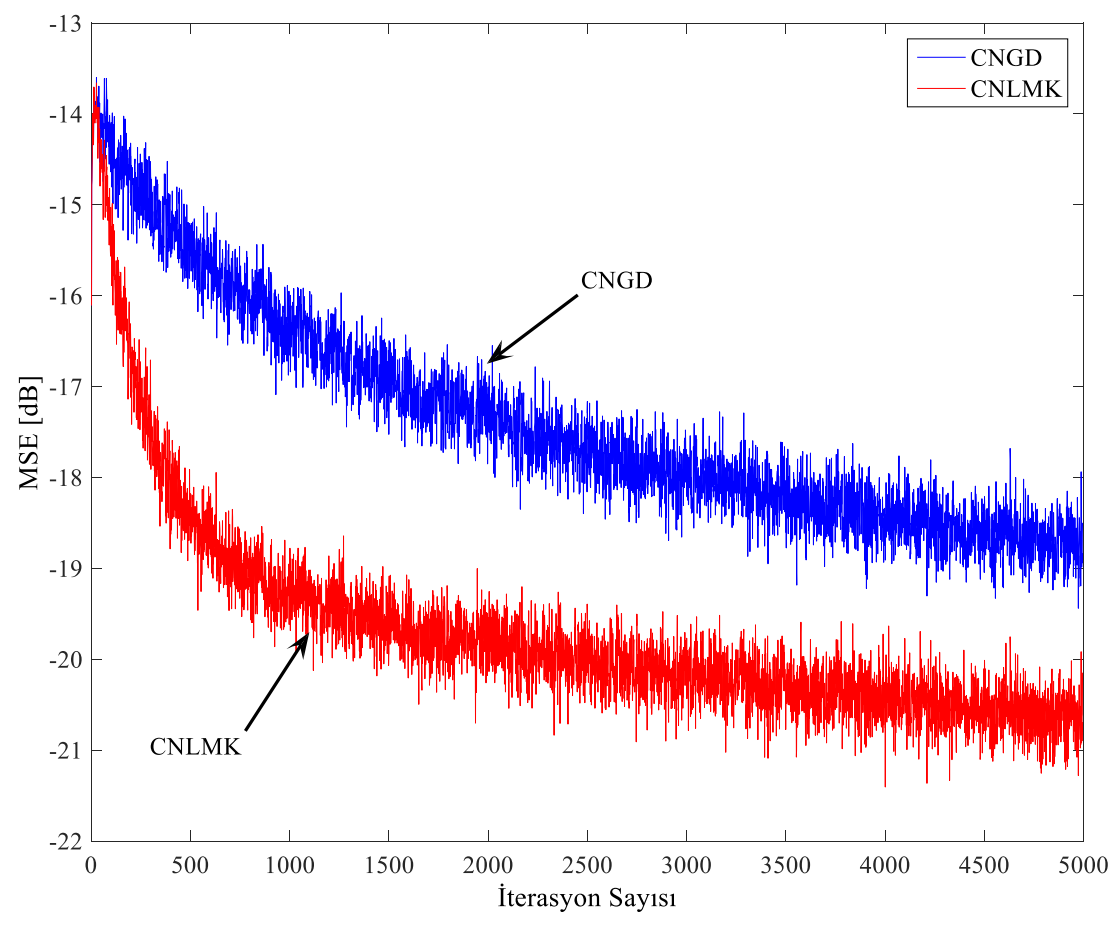

Şekil 6. Benzetimde kullanılan karmaşık-değerli AR sinyali için CNLMK ve CNGD algoritmalarının MSE (dB) başarımları

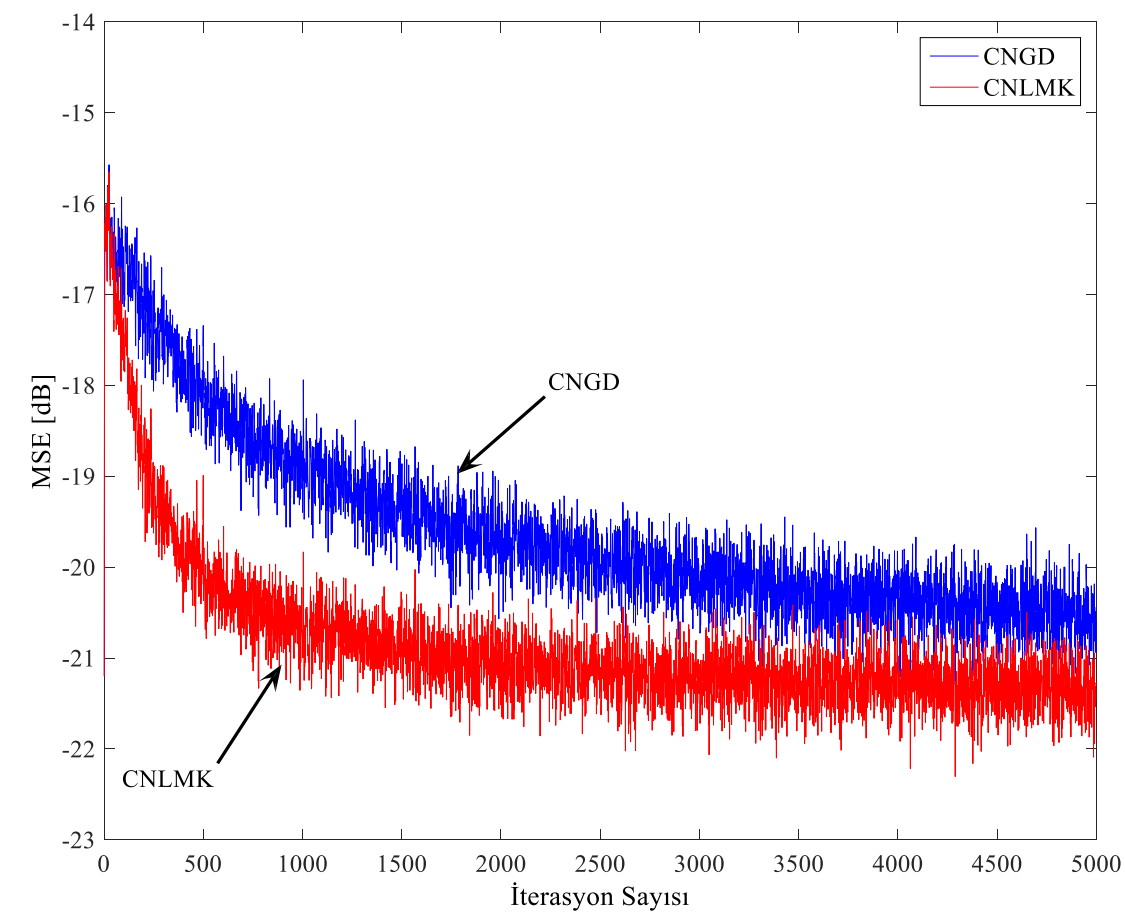

Şekil 7. Benzetimde kullanılan karmaşık-değerli ARMA sinyali için CNLMK ve CNGD algoritmalarının MSE (dB) başarımları 


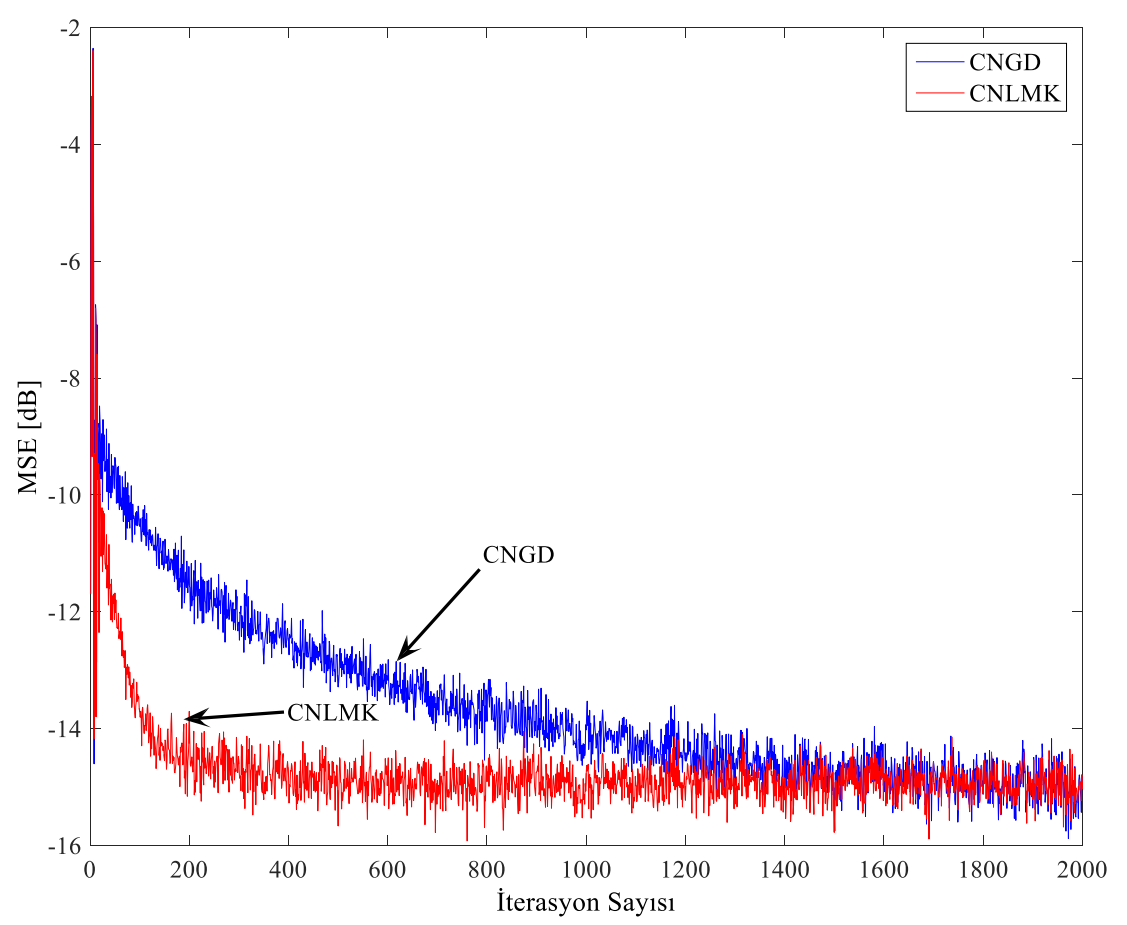

Şekil 8. Benzetimde kullanılan karmaşık-değerli Ikeda sinyali için CNLMK ve CNGD algoritmalarının MSE (dB) başarımları

\section{SONUÇ (CONCLUSION)}

Bu çalışmada, karmaşık-değerli sinyallerin adaptif tahmini için yeni bir tam karmaşık doğrusal olmayan CNLMK algoritması önerilmiştir. Önerilen algoritmanın tasarımında, öncelikle hata sinyalinin kurtosisi, maliyet fonksiyonu olarak karmaşık düzlemde ifade edilmiş ve doğrusal olmayan filtre yapısı dikkate alınarak minimize edilmiştir. Bu sayede karmaşık düzlemde çalışma kabiliyetine sahip doğrusal olmayan FIR adaptif filtre algoritması karmaşık-değerli sinyallerin işlenebilmesi için tasarlanmıştır. Tasarlanan algoritmanın başarımı, CNGD algoritması ile dört farklı karmaşık-değerli sinyal tahmini üzerinde karşılaştııılmıştır. Benzetim sonuçları, CNLMK algoritmasının MSE (dB) ve tahmin kazancı (dB) açısından CNGD algoritmasını geride bıraktığını göstermiştir.

\section{KAYNAKLAR (REFERENCES)}

[1] S. Haykin, Adaptive Filtering Theory, Englewood Cliffs, NJ: Prentice-Hall, 1995.

[2] D. P. Mandic, S. L. Goh, Complex Valued Nonlinear Adaptive Filters: Noncircularity, Widely Linear and Neural Models, Wiley, 2009.

[3] B. Widrow, J. M. McCool, M. Ball, The complex LMS algorithm, Proceedings of the IEEE, 63 (1975) 719-720.

[4] Y. Xia, C. C. Took, D. P. Mandic, An augmented affine projection algorithm for the filtering of noncircular complex signals, Signal Processing, 90 (2010) 1788-1799.

[5] B. Jelfs, D. P. Mandic, S. C. Douglas, An adaptive approach for the identification of improper complex signal, Signal Processing, 92 (2012) 335-344. 
[6] E. C. Mengüç, N. Acır, An augmented complex-valued Lyapunov stability theory based adaptive filter algorithm, Signal Processing, 137 (2017) 10-21.

[7] E. C. Mengüç, N. Acır, Complex-valued least mean Kurtosis adaptive filter algorithm, IEEE 24. Signal Processing and Communication Applications Conference, (2016) 325-328.

[8] D. P. Mandic, S. Javidi, S. L., Goh, A. Kuh, K. Aihara, Complex valued prediction of wind profile using augmented complex statistics, Renewable Energy, 34 (2009) 196-201.

[9] O. Tanrikulu, A. G. Constantinides, Least-mean kurtosis: A novel high order statistics based adaptive filtering algorithm, Electronics Letters, 30 (1994) 189-190.

[10] P. I. Hubscher, J. C. M. Bermudez, A model for the behavior of the least mean kurtosis (LMK) adaptive algorithm with Gaussian inputs, Proceeding of the 2002 International Telecommunications Symposium, (2002) 1-4.

[11] N. J. Bershad, J. C. M. Bermudez, Stochastic analysis of the least mean kurtosis algorithm for gaussian inputs, Digital Signal Processing, 54 (2016) 35-45.

[12] L. Lu, H. Zhao, Improved filtered-x least mean kurtosis algorithm for active noise control, Circuits, Systems and Signal Processing, 36 (2017) 1586- 1603.

[13] J. K. Hwang, Y. P. Li, A gradient-based variable step-Size scheme for kurtosis of estimated error, IEEE Signal Processing Letters, 17 (2010), 31-334.

[14] Z. Tang, Z. Yan, Y. Yao, Blind multiuser detector based on LMK criterion, Electronics Letters, 35 (1999) 267-268.

[15] E. C. Mengüç, N. Acır, An augmented complex-valued least-mean kurtosis algorithm for the filtering of noncircular signals, IEEE Transactions on Signal Processing, 66 (2018) 438-448.

[16] E. C. Mengüç, Novel quaternion-valued least-mean kurtosis adaptive filtering algorithm based on the GHR calculus, IET Signal Processing, 12 (2018), 487-495.

[17] J. Chambers, O. Tanrikulu, A. G. Constantinides, Least mean mixed-norm adaptive filtering, Electronics Letters, 30 (1994) 1574-1575.

[18] S. C. Pei, C. C. Tseng, Least mean p-power error criterion for adaptive FIR filter, IEEE Journal on Selected Areas in Communications, 12 (1994) 1540-1547.

[19] Y. Zou, S. C. Chan, T. S. Ng, Least mean M-estimate algorithms for robust adaptive filtering in impulse noise, IEEE Transactions on Circuits and Systems II: Analog and Digital Signal Processing, 47 (2000) 1564-1569.

[20] P. I. Hubscher, J. C. M. Bermudez, V. E. Nascimento, A mean square stability analysis of the least mean fourth adaptive algorithm, IEEE Transactions on Signal Processing, 55 (2007) 4018-4028.

[21] A. I Hanna, D. P. Mandic, Adaptive normalized nonlinear gradient descent algorithm for complexvalued nonlinear adaptive filters, IEEE Transactions on Signal Processing, 50 (2003) 2540-2549.

[22] The McMaster IPIX radar sea clutter database (2018): http://soma.ece.mcmaster.ca/ipix. 